\title{
Diagnosis of composite materials in aircraft applications: towards a UAV active thermography inspection approach
}

\author{
M. Alhammad ${ }^{1}$, N.P. Avdelidis ${ }^{1,2^{*}}$, S. Deane ${ }^{1}$, C. Ibarra-Castanedo ${ }^{2}$, S. Pant ${ }^{3}$, M. Ahmadi², \\ M. Genest ${ }^{3}$, A. Zolotas ${ }^{1}$, L. Zanotti-Fragonara ${ }^{1}$, J. Valdes ${ }^{3}$, X.P.V. Maldague ${ }^{2}$ \\ ${ }^{1}$ School of Aerospace, Transport and Manufacturing, Cranfield University, UK. \\ ${ }^{2}$ Universite Laval, Computer Vision and Systems Laboratory, Department of Electrical and \\ Computer Engineering, Quebec City, G1V 0A6, Canada. \\ ${ }^{3}$ National Research Council Canada, Ottawa, ON K1A 0R6, Canada.
}

\begin{abstract}
Diagnosis and prognosis of failures for aircrafts' integrity are some of the most important regular functionalities in complex and safety-critical aircraft structures. Further, development of failure diagnostic tools such as Non-Destructive Testing (NDT) techniques, in particular, for aircraft composite materials, has been seen as a subject of intensive research over the last decades. The need for diagnostic and prognostic tools for composite materials in aircraft applications rises and draws increasing attention. Yet, there is still an ongoing need for developing new failure diagnostic tools to respond to the rapid industrial development and complex machine design. Such tools will ease the early detection and isolation of developing defects and the prediction of damages propagation; thus allowing for early implementation of preventive maintenance and serve as a countermeasure to the potential of catastrophic failure. This paper provides a brief literature review of recent research on failure diagnosis of composite materials with an emphasis on the use of active thermography techniques in the aerospace industry. Furthermore, as the use of unmanned aerial vehicles (UAVs) for the remote inspection of large and/or difficult access areas has significantly grown in the last few years thanks to their flexibility of flight and to the possibility to carry one or several measuring sensors, the aim to use a UAV active thermography system for the inspection of large composite aeronautical structures in a continuous dynamic mode is proposed.
\end{abstract}

Keywords: Failure diagnosis, aircraft applications, UAV inspections, thermography, imaging.

\section{INTRODUCTION}

Composite materials have been utilized for various machining and industrial processes since last decades until now. Composite materials are so called because they are formed from two or more materials, one of which, the fibre, is scattered in a continuous matrix phase. The two materials work together so that they provide different material properties from the properties of the original elements on their own [1]. Though most airplanes today are made out of aluminum, composite materials such as those materials made from carbon fibres, glass fibres, and Kevlar, to name few, are widely used in the aircraft industry. For instance, glass fibres were first employed in aircraft, by Boeing, in the 1950s. Also, Boeing 787 Dreamliner was the first commercial airplane to be built from more than 50\% composite materials, mostly carbon fibre composites. Since 2014 or so, there have also been a noticeable increase in the use of composite materials in aircraft industry compared with the other materials, as shown in figure 1 . The greatest advantage of composite materials usage is the lower-weight planes. For instance, they are stronger, yet lightweight than aluminium. This weight reduction will lead to more fuel-efficient planes that will require less fuel to propel themselves forward. High strength-to-weight ratio, also known as specific strength, is another advantage of the composites and as a result are incredibly stronger than the other metals used in aircraft manufacturing. Hence, they can resist compression loads and do not easily break under tension conditions.

\footnotetext{
${ }^{*}$ Correspondance e-mail: np.avdel@cranfield.ac.uk
} 
Composite materials are also insusceptible to corrosion due to harsh chemical components, and their resistant to many highly reactive chemicals. Further, they can incredibly handle the exposure to severe weather conditions including a wide variation in temperature. This is very important in the aircraft industry as the most essential parts of any airplane made out of composite materials will neither grow nor shrink or change as a result of varying environmental conditions. Design flexibility represents another big advantage of composite materials over the other metals. Composites can be formed and/or made into just about the required shape. For instance, a single oddly shaped piece of composite can replace many pieces made out of other materials. This helpful characteristic lead eventually not only to cut down the maintenance cost but also reduce the total cost over the lifetime of planes.

Used Materials in Aircraft Industry 1985-2014

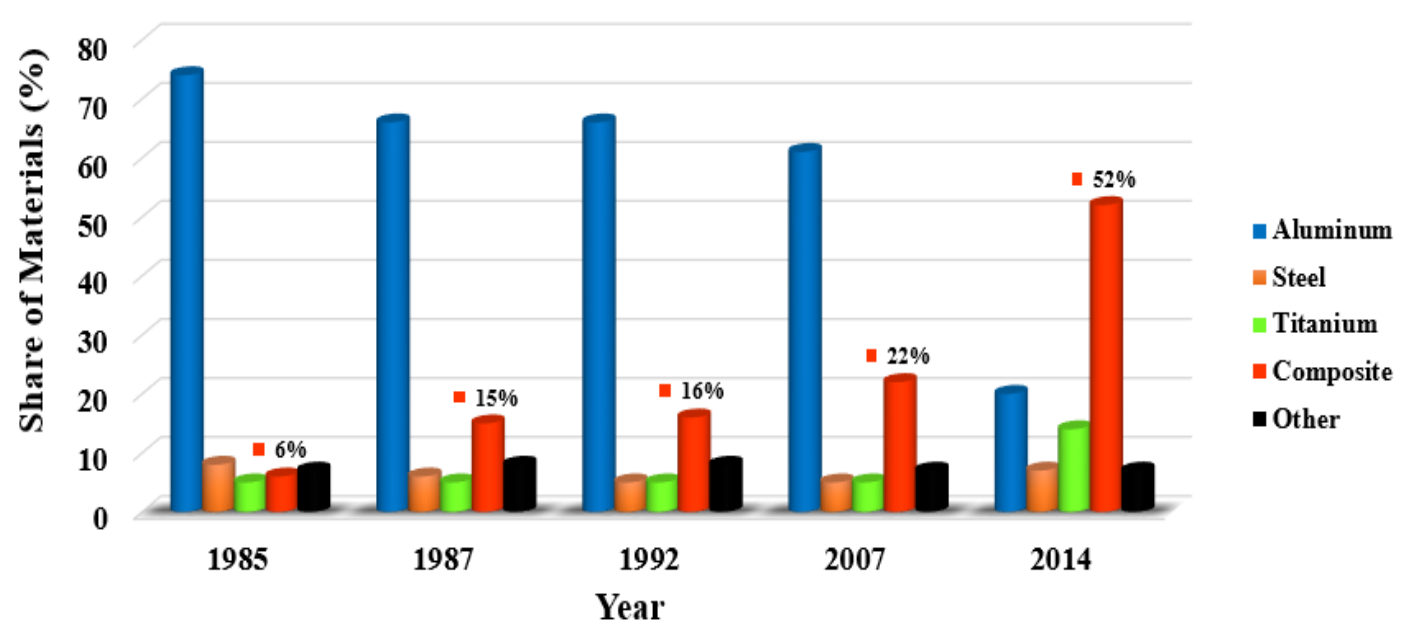

Figure 1. Used Materials in Aircraft Industry 1985-2014 [2].

Nevertheless, composite materials have revolutionized the aviation industry, they have, however, their own limitations compared with the alternative materials; higher initial cost for aircraft and component manufacturers compared with the other metals is perhaps the biggest one. This high initial cost is mainly attributed to the high price of fibres and the complicated manufacturing processes. Another drawback is that the prediction of failure time for the internal structure of any composite aircraft piece cannot easily be made. This, in turn, leads to very costly and difficult damage inspections. Also, during the inspections, delamination may occur, layers of composite separate, as a result of an impact to the composite piece. As consequence of this, water may penetrate to the delaminated composite piece, causing serious problems, in particular, when water freezes and thaws. Unlike, for instance, aluminium, most of standard composite materials used in aircraft industry do not conduct electricity, and therefore they cannot prevent, for example, lightning from being directed to the fuel tanks. However, it was reported that composites were integrated with wire mesh to fix this problem; this method was first used in 787 aircrafts. The existence of resin in the manufactured composite materials is reported as one of the causal factors behind the catastrophic failure. At temperatures below $65^{\circ} \mathrm{C}$, resin weakens the composites and yet it may also lead to aircraft failure when temperatures reach even as above as $150^{\circ} \mathrm{C}$.

As it can be seen the use of composites in aircraft industry does present some engineering and maintenance challenges. This involves damage inspections and diagnosis that may lead to costly manufacturing and repairs. With more and more commerce and business being conducted every day in this field, analysis of data for detecting and repelling damage of composites is paramount to every aircraft company. To directly tackle and respond to the lack of the engineering improvement for the damage inspection techniques for composites in aircraft industry, off the shelf, tremendous work over a number of recent years was undertaken. Though several attempts were made to summarize this intensive number of published works it is still unfortunately scattered here and there. 


\section{FAILURE INSPECTIONS FOR COMPOSITE MATERIALS}

Failure inspections or failure diagnosis can be defined as the process of analyzing physical parameters, associated with the structure, for the purpose of determining structure integrity [3]. Failure diagnosis involves the prediction of damage onset on a structure when subjected to loads and environmental conditions. Failure may consist permanent damages such as structural deformations (plastic deformation), or lack of functionality [3]. It is worth mentioning that crack/damage onset does not mean the final form or catastrophic failure of the structure. To keep, for instance, aircrafts function at optimal levels, condition monitoring of structural components (e.g. composites) is important because any wear, if is not caught in time, will often progress to more serious damage affecting the adjacent parts. A short review of NDT and other failure diagnosis methods, which are possibly viable for composites in aircraft applications, to evaluate a specific type of damage identification is presented.

NDT refers to a method of detecting internal flaws in engineering materials without breaking them [4-5]. It can also be defined as the use of non-invasive technique to determine the integrity of a material, component or structure or quantitatively measure some characteristics of an object. It is very broad interdisciplinary field that plays a vital role in analysing the properties and parameters of any material [5]. Advantages of NDT methods include:

- Analysis of parts can be done without breaking them.

- Cost saving procedure.

- Improve the quality of production.

- Saves time in product evaluation.

- Evaluation can be done at manufacturing stage or in service stage.

- $\quad$ Portable mode of inspection.

- Surface defects and inside defects can be easily evaluated.

NDT techniques can be classified in many ways such as Conventional and Advanced methods, Passive and Active techniques or Contact and Non-Contact methods., etc., to name a few. Conventional techniques are the NDT methods that have well-established and fully matured over the past decades. Yet, they are described as well-documented techniques in terms of codes, standards, and best practices. In contrast, advanced methods are very limited in use as they tend to be less understood due to either uncertain advantages or limitations, lack of laborious qualification criteria, or little to no industry codification.

With the passive approach, NDT methods, e.g. acoustic emission, are used to investigate different materials/structures by reflecting their internal energy using proper physical fields, whilst in the case of the active approach an external stimulus source (forced physical field such as heat, force, cold air gun etc.) is used. Contact NDT type is commonly used in construction industry where, for instance, transducers are directly attached to the materials or structures under test, e.g. Ultrasonic testing. In the Non-contact methods, data are collected without the need for a direct accessibility to the surface of the structure being tested, e.g. thermography test.

Classification NDT techniques based on the category of contact or non-contact methods is presented in the following table.

Table 1 Contact and Non-Contact NDT Methods

\begin{tabular}{cc}
\hline Contact Methods & Non-Contact Methods \\
\hline Traditional Ultrasonic Testing & Air-Coupled Ultrasonic Testing \\
Acoustic Emission & Radiography Testing \\
Eddy Current Testing & Thermography \\
Magnetic Testing & Shearography \\
Electromagnetic & Holography \\
Liquid Penetrant & Visual Inspection \\
\hline
\end{tabular}


Attempts were made to evaluate the integrity conditions of composites in aircraft applications using NDT techniques. In the literature, a number of case studies for failure diagnosis in composite materials are well described. An extensive review of the use of NDT methods for material inspections was made by Gholizadeh [6]. Another review for nondestructive testing and evaluation (NDT\&E) methods applied in inspections of structural aircraft components was presented in [7]. In this review, applications of Visual and Penetrant Inspection, Tap-Testing, Eddy Current Inspection, Shearography, Thermography, Acoustic Emission Testing, and Ultrasonic Inspection were highlighted. More reviews on the use of NDT techniques for the composite materials, were also well documented [8-9]. Towsyfyan et al. [10] highlighted in sufficient details the success and challenges of different certified NDT methods that are applicable for damage detection and characterization in composite laminates used in the aircraft primary and secondary structures. Ehrhart et al. [11] summarized the NDT techniques that are, in particular, possibly viable for adhesively bonded composite materials. The summary focused on typical defects and quality management of composites, used in aerospace industry and substrates' applications, throughout the product life cycle.

\section{APPLICATIONS OF THERMOGRAPHY FOR AIRCRAFT COMPOSITES}

Detection of defects using Infrared Thermography (IRT) has been the topic of investigation by several researchers [1215], employing different approaches in order to perform aircraft composites inspection and evaluation. The selection of the thermal excitation sources has to be given a paramount of importance. The reason behind this is that for the selection of proper thermal stimulation sources, factors such as clearance and accessibility of the samples, available power consumption, inspection cost, limitations, etc., to name a few, are considered. This has represented one interesting area of further research until today's date, and hence a number of studies have been undertaken to investigate the potential for improving/developing upon the existing thermal excitation methods.

To directly tackle and respond to the lack of this engineering improvement, some studies have shredded light on the use of Microwave Thermography (MWT) and Material-Based Thermography approaches. To generate the required heat, the former basically employs electromagnetic radiation microwaves with high frequencies ranging from $300 \mathrm{MHz}$ to 30 $\mathrm{GHz}$ [16]. The latter is based on either exploiting some specific properties of samples under examination and accordingly assess the internal health integrity; this method is known as a Direct Material-Based Thermography (DMT) or adding some thermos-resistive components to the composites at the manufacturing stage, which will work as an internal thermal stimulation when thermography tests are performed; this approach is known as an Indirect MaterialBased Thermography (DMT) [17]. It is worth mentioning that the application of MWT method in aerospace industry has only been introduced in the recent years. And there are other several promising approaches that have also been used in aircraft composites inspection, apart from pulsed thermography (PT), lock-in thermography (LT) and vibro thermography (VT), that are the most common ones: laser-line thermography (LLT) or laser-spot thermography (LST), ultrasonic stimulated thermography (UST), and eddy current stimulated thermography (ECST), to name a few.

From there, several attempts were made to develop new methods to improve the overall performance of thermographic techniques. For example, for better image contrast, and accordingly improved damage identification, Thermographic Signal Reconstruction (TSR) has been applied to Step Heating Thermography (SHT) and Long Pulse Thermography (LPT) for damage inspections in composites [18]. Furthermore, Frequency Modulated Thermography (FMT) method (time domain and frequency domain representations) to analyse an acquired data from CFRP samples has shown that the use of frequency domain (phase) method led to improvement in resolution and high sensitivity for damage detection [19].

As it can be seen that the literature, indeed, abounds with numerous attempts made not only to improve the existing thermography methods for the evaluation of aircraft composites but also to develop new techniques. However, these attempts were not only limited to the improve and/or develop new inspection tools rather they show that there are as well some studies have applied some well-established techniques for NDT diagnostics. For instance, assess the health status of aircraft composites using transient thermal NDT and identify failure modes such as impact damage, drilling induced defects, delaminations, etc have been successfully investigated. Real time monitoring has been achieved and from there the employment of specifically designed robotic (ground and/or aerial) platforms for autonomous infrared thermographic inspection of aircraft composites is the main aim in order to provide a reliable, rapid and robust tool for detection and characterization of different damages within aircraft materials. 


\section{UAV ACTIVE THERMOGRAPHY INSPECTION APPROACH}

Large structures can be inspected using a dynamic configuration. This is the so-called line scan thermography (LST), where the camera and the heat source move in tandem (the camera records thermograms right after heating) with respect to the surface of the component, which is normally static while being inspected. This can be performed for example by mounting the camera and source in a robot or a 2-axis actuator. LST allows to inspect large and/or complex shaped components faster than classical static IRT. It is an excellent option for quality control during production.

For in-service inspection, the ideal situation would be to inspect an aircraft without the need of removing any component. The LST system would require in this case a huge robotic arm or several smaller robots properly installed and properly distributed to cover all the areas of the plane that need to be inspected. Alternatively, a dynamic system moving "freely" around the aircraft and performing the inspection of all the areas of interest in a fast and effective manner can be conceived, this is where an UAV system becomes interesting.

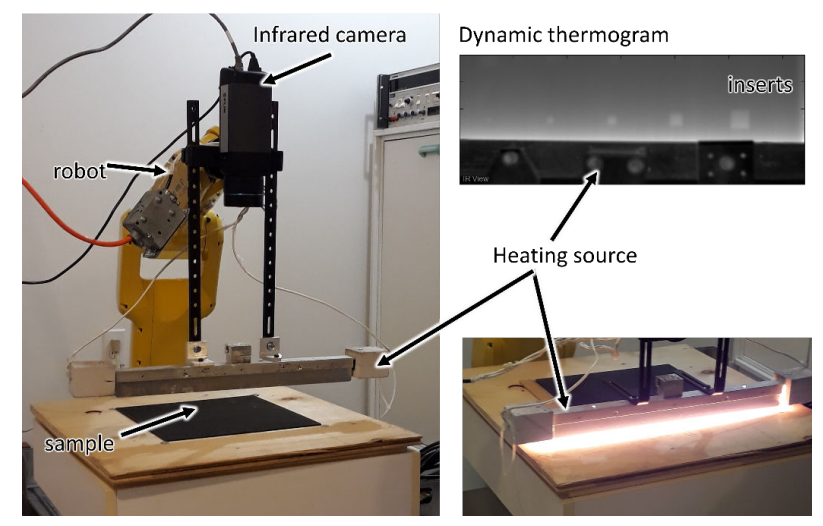

Figure 2. Line Scan Thermography (LST) approach.

UAVs and IRT are a perfect match for the contactless survey of thermal phenomena of either large and/or otherwise inaccessible areas, or to ease the inspection of large structures of difficult access. The use of UAV-IRT systems have been explored in the examination of numerous applications. In all these cases, the passive approach has been employed, i.e. the observation of thermal phenomena without the use of external energy stimulation, with the assumption that the features of interests (plants, building materials, PV cells) will naturally produce thermal gradients that can be clearly isolated from the scene or background. This assumption is valid in many cases, such as building inspection (e.g. detection of thermal bridges, air leakages, moisture or humidity); precision agriculture (e.g. monitor nutriments levels or lack of water in crop fields; quality assessment of large structures (e.g. inspection of photovoltaic panels farms, wind turbines); or for surveillance applications (e.g. people or animal surveillance).

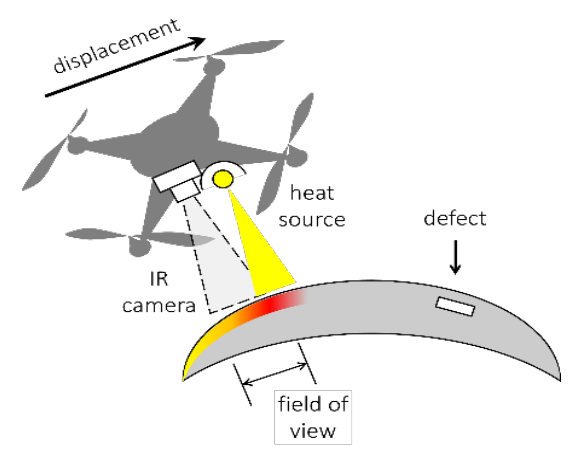

Figure 3. Schematic of UAV IRT Validation for the inspection of actual aircraft. 
There are however some problems/limitations to take into consideration: the drone has to carry the IR camera, possibly (ideally) a visible camera and a heat source. Both cameras can be feed by battery with decent autonomy. The heating source, however, consumes considerable amounts of energy and would be difficult to operate for long periods through a battery. One possibility is to separate the heat source from the drone and operate it independently from a grounded power supply. Another possibility is to have the UAV permanently connected to a power cable, which will assure a constant power supply. In the case of integrating the thermal excitation source to the UAV, it is important to analyse the weight and power consumption that will allow you to understand the UAV's flight time and trajectory. The other main objectives are as follows:

- To understand the effective thermal transfer from source to target.

- To understand the thermal stability characteristics of the source.

- To establish design / development direction of active heat source.

To incorporate the proposed lamp a housing needs to be developed, which is electrically safe and impact resistant. The first method used was to design a bespoke mounting unit which will hold the socket and the bulb onboard the UAV. An efficient way to manufacture complex shapes for rapid prototyping is by using a commonly used 3D printing method known as fused deposition modelling (FDM). This method uses thermoplastic, which is a material than can be cooled shaped and heated without any change in their chemical or mechanical properties. At an elevated temperature the material can be moulded into complex required shaped.

\section{MACHINE LEARNING APPROACH}

Machine learning algorithms have been employed to improve the feasibility of IRT for the inspection of composites. Example of this was the research work undertaken by Saeed et al. [20]. Artificial Neural Network (NN) was integrated with a line-scan thermography to detect different defects depth. This configuration was applied to specifically designed CFRP coupons with embedded and flat bottom holes' defects. Prior to the experimental analysis using the proposed NN method, a Multiphysics FEM simulation model of the inspection process was run to generate some training datasets for the assessment of the developed NN structure in a deterministic (noise free) environment. It was concluded that the detection accuracy of the proposed NN for the simulated data and the experimental data was $97 \%$ and $90 \%$ respectively.

In order to achieve a successful and accurate training through a machine learning technique, thousands of annotated training samples are required. This is a relatively straightforward procedure in areas such as biomedical image processing. However, in the case of NDT, especially in the aerospace sector, it is more challenging to prepare such a huge data base.

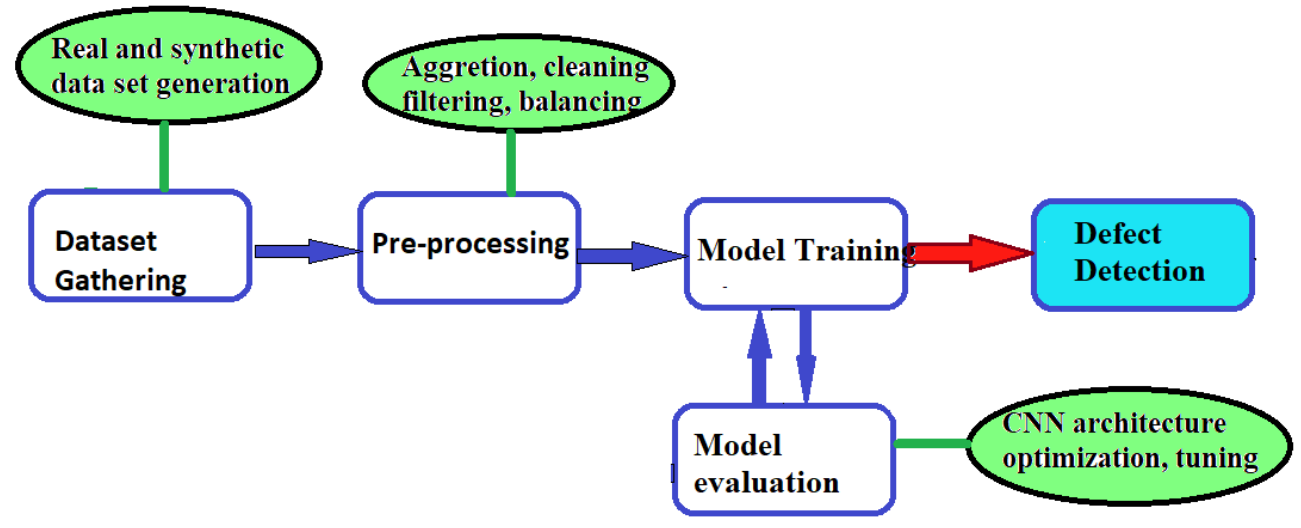

Figure 4: Main steps for defect classification based on machine learning. 
The main steps for the classification of the defects from the thermographic inspections based on machine learning, is also proposed (see figure 4) and intended to be used once sequences of images are acquired from the UAV thermography inspections. U-Net is a deep learning Convolutional Neural Network (CNN) based technique that has been proven reliable and efficient for a wide variety of applications. The U-Net structure consists of a contracting path to capture context and a symmetric expanding path that enables precise localization. It has been shown that by use of this architecture the network trained with just few images.

\section{CONCLUSIONS}

This paper provided a brief literature review of recent research on failure diagnosis of aircraft composites with an emphasis on the use of active thermography techniques. Furthermore, as the use of UAVs for the remote inspection of large and/or difficult access areas has significantly grown in the last few years thanks to their flexibility of flight and to the possibility to carry one or several measuring sensors (including thermal infrared systems), the aim to develop a UAV active thermography approach for the inspection of large aircraft composite structures in a continuous dynamic mode was proposed. Next steps include the use of this UAV inspection approach on aerospace composites and in parallel the machine learning approach presented developed fully from the data of the UAV thermography inspections, aiming to autonomous and effective in-service aircraft inspections. This will contribute to reducing the time and cost of NDT composite inspections.

\section{REFERENCES}

1. Kapadia, A. Best Practice Guide, Non-Destructive Testing of Composite Materials. https://avaloncsl.files.wordpress.com/2013/01/ncn-best-practice-ndt.pdf, (accessed April 2020).

2. Statista Distribution of composite materials used in commercial aircraft manufacturing worldwide from 1985 to 2014. https://www.statista.com/statistics/954913/share-composites-in-aircraft-design/\#statisticContainer.

3. Makhlouf, A. S. H.; Aliofkhazraei, M., Handbook of Materials Failure Analysis with Case Studies from the Construction Industries. Vol. 6. Butterworth-Heinemann Imprint Elsevier Science \& Technology Books, Elsevier Science \& Technology Distributor: San Diego, Oxford, 2018; p. 560 p.

4. Wang, B.; Zhong, S.; Lee, T.-L.; Fancey, K. S.; Mi, J., Non-destructive testing and evaluation of composite materials/structures: A state-of-the-art review. Advances in Mechanical Engineering 2020, 12 (4), 1687814020913761.

5. Prakash, R., Non Destructive Testing Techniques. New Academic Science: Kent, 2009; p. 140 p. ill.

6. Gholizadeh, S., A review of non-destructive testing methods of composite materials. Procedia Structural Integrity 2016, 1, 50 - 57.

7. Wronkowicz-Katunin, A., A Brief Review on NDT\&E Methods For Structural Aircraft Components. Fatigue of Aircraft Structures 2018, 2018 (10), 73.

8. Akademia Baru, P.; Khattak, M.; Mukhtar, A.; Shahid, I.; Sufian, S. M., A Review on Application of Non Destructive Techniques on Composites. 2016, 12-21.

9. Vavilov, V. P.; Burleigh, D. D., Review of pulsed thermal NDT: Physical principles, theory and data processing. NDT \& E International 2015, 73, 28-52.

10. Towsyfyan, H.; Biguri, A.; Boardman, R.; Blumensath, T., Successes and challenges in non-destructive testing of aircraft composite structures. Chinese Journal of Aeronautics 2020, 33 (3), 771-791.

11. Ehrhart, B.; Valeske, B.; Bockenheimer, C., 9 - Non-destructive evaluation (NDE) of aerospace composites: methods for testing adhesively bonded composites. In Non-Destructive Evaluation (NDE) of Polymer Matrix Composites, Karbhari, V. M., Ed. Woodhead Publishing: 2013; pp 220-237.

12. Ciampa, F.; Mahmoodi, P.; Pinto, F.; Meo, M., Recent Advances in Active Infrared Thermography for NonDestructive Testing of Aerospace Components. Sensors 2018, 18 (2), 609.

13. Katunin, A.; Dragan, K.; Dziendzikowski, M., Damage identification in aircraft composite structures: A case study using various non-destructive testing techniques. Composite Structures 2015, 127, 1-9.

14. Péronnet, E.; Pastor, M.; Huillery, R.; Dalverny, O.; Mistou, S.; Welemane, H., Non-Destructive Investigation of Defects in Composite Structures by Three Infrared Thermographic Techniques. In 15th International Conference on Experimental Mechanics, Porto, Portugal 2012.

15. Avdelidis, N. P.; Hawtin, B. C.; Almond, D. P., Transient thermography in the assessment of defects of aircraft composites. NDT \& E International 2003, 36 (6), 433-439. 
16. Foudazi, A.; Donnell, K. M.; Ghasr, M. T. In Application of Active Microwave Thermography to delamination detection, 2014 IEEE International Instrumentation and Measurement Technology Conference (I2MTC) Proceedings, 12-15 May 2014; 2014; pp 1567-1571.

17. Yang, R.; He, Y., Optically and non-optically excited thermography for composites: A review. Infrared Physics \& Technology 2016, 75, 26-50.

18. Almond, D. P.; Angioni, S. L.; Pickering, S. G., Long pulse excitation thermographic non-destructive evaluation. NDT \& E International 2017, 87, 7-14.

19. Arora, V.; Siddiqui, J. A.; Mulaveesala, R.; Muniyappa, A., Pulse Compression Approach to Nonstationary Infrared Thermal Wave Imaging for Nondestructive Testing of Carbon Fiber Reinforced Polymers. IEEE Sensors Journal 2015, 15 (2), 663-664.

20. Saeed, N.; Abdulrahman, Y.; Amer, S.; Omar, M. A., Experimentally validated defect depth estimation using artificial neural network in pulsed thermography. Infrared Physics \& Technology 2019, 98, 192-200. 


\title{
Diagnosis of composite materials in aircraft applications: towards a UAV active thermography inspection approach
}

\author{
Alhammad, Muflih \\ Society of Photo-Optical Instrumentation Engineers (SPIE)
}

Alhammad M, Avdelidis NP, Deane S,et al., (2021) Diagnosis of composite materials in aircraft applications: towards a UAV active thermography inspection approach. In: SPIE Defense + Commercial Sensing, Proceedings Volume 11743, Thermosense: Thermal Infrared Applications XLIII, 12-17 April 2021, Virtual Event

https://doi.org/10.1117/12.2586064

Downloaded from Cranfield Library Services E-Repository 